http://jmscr.igmpublication.org/home/ ISSN (e)-2347-176x ISSN (p) 2455-0450

crossref DOI: https://dx.doi.org/10.18535/jmscr/v8i11.71

Journal Of Medical Science And Clinical Research

\title{
Paediatric Cataract Observation \& Results in my Study
}

\author{
Authors \\ Dr N. Suhasini M.S. ${ }^{1}$ FIGS, Dr V. Suryanarayana M.S., D.O². \\ ${ }^{1}$ Asst.Prof-KIMS \& RF, Amalapuram \\ ${ }^{2}$ Retd. Prof, REH, Visakhapatnam
}

\begin{abstract}
Aim: To describe \& analyze the current techniques utilized in the management of paediatric cataracts, their visual outcome \&complications of cataract surgery.

Materials \& Methods: A retrospective study of 68 subjects who underwent cataract surgery during 2007-08 in Govt. REH. PMMA intra ocular Lens $(12 \mathrm{~mm})$ was implanted in the bag for children more than 2 years. Intraocular lenses were not implanted for children aged less than 2 years. Optical correction was given.

Results \& Discussion: For aphakic patients, contact lenses along with spectacles are prescribed for near \& to correct aniseikonia (or) only aphakic spectacles are prescribed. For pseudophakic patients, reading spectacles are prescribed.

Conclusion

1. Cataract is more common in male children (87\%) compared to females.

2. Cataract is more common in lower socioeconomic status in the age group of 6-9 Yrs. (43.75\%)

3. Bilateral congenital and developmental cataracts of Zonular type is found to be more common in our study.

4. Unilateral cataracts, Bilateral cataracts where the management has been delayed or associated nystagmus and strabismus has set in, have a poor visual outcome.

5. Primary posterior chamber Intraocular lenses are recommended for children 2 Yrs. of age and older.

6. Most common complication is posterior capsular opacification, managed with YAG laser capsulotomy in most of the cases.

7. Early surgery combined with appropriate refractive correction and aggressive amblyopia therapy provides good visual outcome.

Keywords: PMMA-Poly Methyl Methacrylate; REH:Regional Eye Hospital.
\end{abstract}

\section{Management of Paediatric Cataract}

The management of cataract in the child depends upon several factors: the child's age; the extent of visual loss; whether or not there is an associated illness or coexisting eye disease; and if the cataract affects one or both eyes.

\section{Surgery}

Intraocular lens (IOL) implantation is becoming an increasingly accepted procedure in young children and infants. Awareness of the rate of myopic shift which takes place in the developing eye, and the use of biometry helps to predict appropriate IOL powers to try to ensure eventual 
emmetropia. However, final refraction is variable, such that emmetropia in adulthood cannot be guaranteed, as there are insufficient long-term studies. Whether or not an IOL is inserted will depend on the age of the child (recently in some centre IOLs are inserted into the eyes of babies as young as four weeks of age), whether or not there is any underlying ocular pathology in which IOL implantation is contra-indicated. In some instances, for example, in remote areas and in less industrialised countries where it is unlikely that the child will be able to be brought back for regular follow-up, IOL implantation is not advisable.

\section{Bilateral Cataracts}

Surgery is indicated when the cataracts are interfering with normal visual development. If the vision is good, then surgery is not indicated, and the child has regular checkups. If there is a significant deterioration in the child's visual acuity at any stage, then surgery is considered .If a child has bilateral dense cataracts, one eye is operated on followed by the second eye one to two weeks later. The first eye is occluded postoperatively until the second eye is operated on, to prevent amblyopia in the un-operated eye. In very young babies, an IOL is not inserted; these babies are given contact lenses one to two weeks post operatively, and if contact lenses are contraindicated, the child is given aphakic spectacles. In some of these children, an IOL may be inserted as a secondary procedure when the eye has grown. In general, an IOL is inserted into the eye of older babies, and depending on the age of the child, an IOL power is selected (according to the biometry) to make that eye appropriately hypermetropic, and hence allow for the myopic shift which takes place in the developing eye; the aim is for emmetropia when the eye is fully grown. In the older child, for example a seven year old child with bilateral cataracts which are beginning to significantly interfere with visual acuity, the eye with the worst visual acuity is operated on, followed by the second eye at least three to four weeks later. All surgeries has potential complications, and the parents are warned about all possible complications pre-operatively (see later). Complications of lens aspiration andIOL implantation include post-operative uveitis, endophthalmitis, glaucoma and retinal detachment. In addition, there is a greater than 90\% risk that the posterior capsule will become thickened within in a year, which requires a further procedure either with laser or surgical capsulotomy to clear the visual axis and restore visual acuity to what it was initially postoperatively. In order to reduce the need for repeated general anaesthetics in order to perform laser or surgery, a primary posterior capsulorrhexis (and limited anterior vitrectomy) may be performed. This involves making a hole in the posterior capsule and removing a small amount of vitreous at the time of the initial cataract surgery. This procedure significantly reduces the development of posterior capsular opacification.

\section{Unilateral Cataract}

In infants with unilateral cataract, the cataract is most likely to be idiopathic and there is usually no associated systemic disease. It is important not to confuse asymmetric bilateral cataract with unilateral cataract and, hence, careful slit lamp examination is essential in all children with cataract, as there may be very subtle changes in one lens and dense cataract in the other. With regard to the management of unilateral cataract, the decision to operate depends on the density of the lens opacity, whether or not there is any associated microphthalmos, and on the parent's motivation to have the surgery performed.

The parents need to be fully informed that surgery alone will not improve their child's vision aiond the management of amblyopia is the key to a successful result. This entails optical correction and many hours of patching the normal eye every day, which as one knows can be extremely difficult in a small infant or toddler, and needs to be maintained for many years. If occlusion is not performed the eye will remain densely amblyopic. It has been reported extensively in the literature 
that children who have had surgery for bilateral cataracts have a much better visual outcome than children who have had surgery for unilateral cataract. Children with unilateral cataract need diligent occlusion therapy of the unoperated eye together with accurate optical correction of the operated eye to obtain a good visual result.

\section{Optical correction post-operatively}

\section{Aphakia}

If the child has not had an IOL inserted and is therefore aphakic, contact lenses are fitted as soon as possible after surgery, regardless of age. This is usually done one to two weeks post-operatively. The baby is refracted and contact lenses are fitted. Contact lenses are relatively safe (Amaya, L., et al., 1990) and they can be used in combination with spectacles for near, and also to correct aniseikonia (Enoch, J.M. and Hamer, R.D.,1983). Rigid gas permeable contact lenses are most suitable for the management of aphakia, as they have a wide range of powers, can correct astigmatism, and are relatively cheap. The disadvantages of contact lens wear are similar to those seen in adults, and include hypoxia and infective keratitis. Parents should be educated about contact lens hygiene and the signs and symptoms of possible complications associated with contact lens wear. All infants and children wearing contact lenses should be kept under constant review at a specialized unit. If contact lenses cannot be fitted (e.g. raised intraocular pressure, external eye disease, dry eye, severe microphthalmos or parents who are unable to cope with contacts), the child is given aphakic spectacles which have the advantage of increasing magnification and, therefore, enhancing the child's acuity, and in can make the eyes of children with microphthalmos look slightly bigger.

\section{Pseudophakia}

To allow for the future growth of the infant eye (i.e myopic shift) an IOL power less than that to result in emmetropia is used. This makes the child hypermetropic (the amount of hypermetropia depends on the age of the child), then as the child's eye grows emmetropia is approached. The child is then given reading spectacles (and a distance prescription if required).

\section{Amblyopia management post-operatively}

The treatment of amblyopia is essential in order to obtain a successful outcome in patients who have had both unilateral and bilateral cataract surgery. Amblyopia is managed by giving the required optical correction and occluding the eye with better visual acuity. The occlusion is performed according to a standardized regime (Tayloe, D.S.I., The Doyne Lecture,1998).It is important to realize that excessive patching of the phakic eye in infants with unilateral aphakia may be associated with increased nystagmus and can have adverse effects on the phakic eye, hence it is imperative that these children are reviewed every two weeks.

\section{Complications of cataract surgery}

Cataract surgery in the child and especially in babies has a higher incidence of complications than those seen after adult cataract surgery. This may be due to several reasons: cataract surgery is technically more difficult in the child's eye, as the tissues are more elastic and behave differently to adult tissues; the child's eye becomes more inflammed than an adult eye in response to an IOL; the very young child's visual systemis still developing and is therefore extremely sensitive to having a defocused image as is the case in aphakia; and a young child is less likely to fully comprehend the importance of keeping dirty fingers away from the operated eye in the immediate post-operative period and, therefore, may rub the eye too vigorously resulting in wound dehiscence and iris prolapse or infection. There have been many reports of the visual outcome and complications of posterior chamber lens implantation in children (Thouvenin, D., 1995).

The most common complications include:

\section{Glaucoma}

Glaucoma may arise in the first few weeks post-operatively, and may present as a watery eye with or without photophobia. The child is usually irritated or just "not his usual self". Some children are 
asymptomatic, hence the importance of regular review with IOP checks postoperatively. Glaucoma may also occur as a late complication years later, and this type of glaucoma can be asymptomatic, therefore all children who have had cataract surgery should be followed up for life.

\section{Uveitis}

All children will develop a certain degree of uveitis post-cataract surgery. This is kept under control with frequent installation of topical steroids, which are given to all children routinely, and are eventually tailed off and discontinued. However, some children (approximately $30 \%$ ) develop inflammation with fibrin formation. This accumulation of fibrin may result in secondary membrane formation blocking the visual axis if not recognized and treated promptly.

\section{Irregular pupil}

An irregularly shaped pupil after cataract surgery may be due to: a) a strand of vitreous coming forwards from the vitreous cavity to the anterior chamber; b) damage to the iris during surgery; and c) iris prolapse, which may be due to accidental trauma to the eye postoperatively and requires urgent attention, as if left untreated that eye may become infected, and even the other eye may be at a risk from sympathetic ophthalmia.

\section{Endophthalmitis}

Bacterial infection of the eye is a devastating complication which can occur in up to $0.4 \%$ of eyes after cataract surgery. It may occur early (i.e. within days to weeks) or late (months to years) post-operatively and presents with a sore painful red eye, and/or reduced vision, and requires urgent hospital admission and treatment

\section{Posterior capsule opacification}

Posterior capsule opacification occurs in up to $90 \%$ of paediatric eyes after lens aspiration when the posterior capsule and anterior vitreous face are left intact. These children, having had a good visual result initially, later present with blurred vision. The thickened posterior capsule is seen using the slit lamp, or simply by examining the red reflex. These children are admitted as a day case and a posterior capsulotomy is performed. Older children can have laser treatment without a general anaesthetic. In some cases, the capsule thickens again and may require surgery to clear the visual axis

\section{Retinal detachment}

Retinal detachment may occur years after cataract surgery, and present with reduced vision which may be preceded by flashes and floaters.

\section{Methodology}

Background: Early diagnosis of congenital cataract is essential in ensuring treatment, together with parental advice and support, can be provided promptly. There is good scope for investigating them using many approaches. Many questions can beeasily addressed through trials and clinical studies. Visual loss in congenital/infantile cataract is mainly due to amblyopia. There is limited information available about the long-term outcomes in children treated with modern surgical techniques. This study is designed to evaluate Paediatric cataracts and its visual out come by using modern techniques among outpatient population of ophthalmology in and around Visakhapatnam Township of Andhra Pradesh.

\section{Objectives}

1) To determine functional results after unilateral and bilateral cataract surgery in children with different aphakic optical correction. 
2) To report treatment of paediatric patients with cataract, and evaluate the efficiency of different surgical interventions.

The specific objectives are:

To examine does cataract extraction combined with primary IOL implantation, compared with cataract extraction without IOL implantation, result in:

- Better overall visual function?

- Reduced risk of aphakic glaucoma?

- Increased risk of significant myopic shift?

Random sampling is used to select a nationally representative sample of adults. $\mathrm{T}$ he study design and protocol are submitted to NTR University of Health Sciences for approval.

Each subject and his parents were interviewed, a brief history of the age of onset of visual loss, involvement of other members of the family, the place of residence, and whether the parents' marriage was consanguineous was recorded. Every subject had their visual acuity measured and underwent autorefraction and fundus/optic disc examination.

An exploration of demographic variables was conducted using regression modelling.

For the study, 60 subjects of cataract, 0- 18 years age groups underwent an extensive ophthalmologic screening examination, including measurements of visual acuity, visual field and fundus photography. Cause of cataract formation in each subject was investigated using all screening information and medical records, to establish the aetiology.

\section{Surgical Technique}

Wound construction was done using self-sealing suture less wound in most cases. Capsular management was obtained in most cases through a central capsulorrhexis measuring around $4 \mathrm{~mm}$. Lensectomy and Anteriorvitrectomy were done in children under 2 years and in traumatic cases where posterior capsule was suspected to be ruptured, a limbal approach was taken for lensectomy and anterior vitrectomy. A primary posterior capsulotomy with or without vitrectomy was performed. PMMA lens $(12 \mathrm{~mm})$ was implanted in the bag for children above 2 years. Intraocular lenses were not implanted for children aged less than 2 years. Children who did not receive IOL implants were rehabilitated postoperatively using spectacles or contact lenses. Patching was done if the cataract was unilateral and amblyopia was present.

The surgical procedures included cataract extraction, intraocular lens implantation, posterior capsulorrhexis and anterior vitrectomy in most of the cases. The visual status of eyes with cataract before and 6 weeks after surgery has been evaluated.

\section{Statistical Analysis}

The results thus obtained are analyzed, and discussed in comparison with the existing studies in the literature. SPSS Version 10.1 software has been used for statistical analysis. Descriptive statistics are computed for baseline demographic, clinical and laboratory features according to aetiology. Frequency tables were generated to reflect the aetiology of cataract and all the values are determined at 5\% significance level. Contingency tables were analyzed through chi squared statistics. Univariate type of parametric type of statistical analysis has been used.

\section{Limitations of the study}

The sample size is small2. Only cases attending Hospital were recruited for the study. 
Table No 13 Pre -operative Visual Acuity of Patients in the given sample

\begin{tabular}{|l|c|c|}
\hline VA & n & Percent \\
\hline could not be assessed & 10 & 20.83 \\
\hline PL+ PR + & 11 & 22.91 \\
\hline HM to $<3 / 60$ & 19 & 39.09 \\
\hline$>3 / 60$ to $<6 / 60$ & 6 & 13.00 \\
\hline$>6 / 60$ & 2 & 4.17 \\
\hline Total & 48 & 100 \\
\hline
\end{tabular}

Preoperative visual acuity is indicated in table No.13 and figure No.10. The vision is only HM to $<3 / 60$ in maximum number cases $39.09 \%$ and least being subjects with vision better than $6 / 60$, amounting to $4.17 \%$. In $20.83 \%$ of children, vision could not be assessed

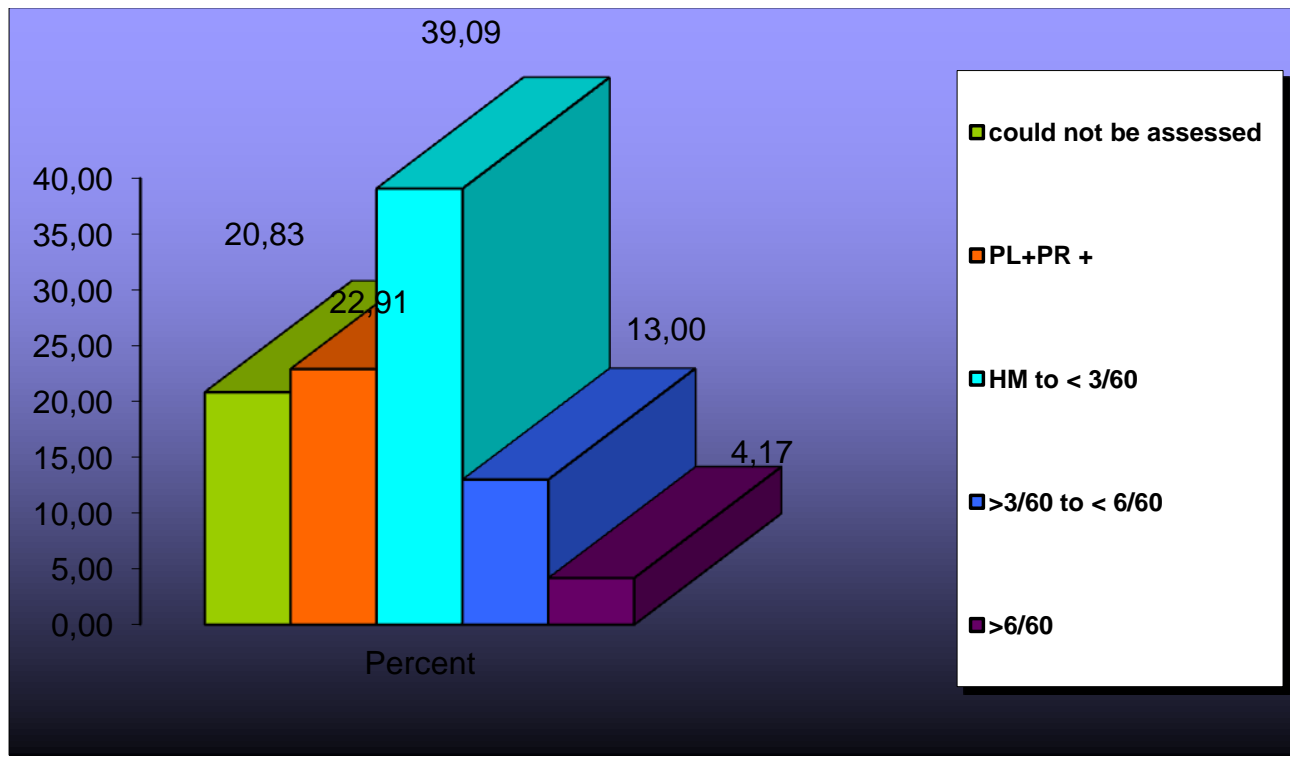

Figure No.10 Pre- Operative Visual Acuity of patients in the given sample

Post Operative visual acuity of patients in the given sample

Table No.17 and figure No.14 show postoperative visual acuity of children in the present sample. Many i.e. $29.17 \%$ of cases could get visual acuity of $<3 / 60$ and another $27.25 \%$ could get visual acuity between $3 / 60$ to $6 / 60$. In $16.67 \%$ of the subjects the vision could get between $6 / 60$ to $6 / 18$. In another major chunk of subjects i.e. $20.83 \%$, vision could not be assessed.

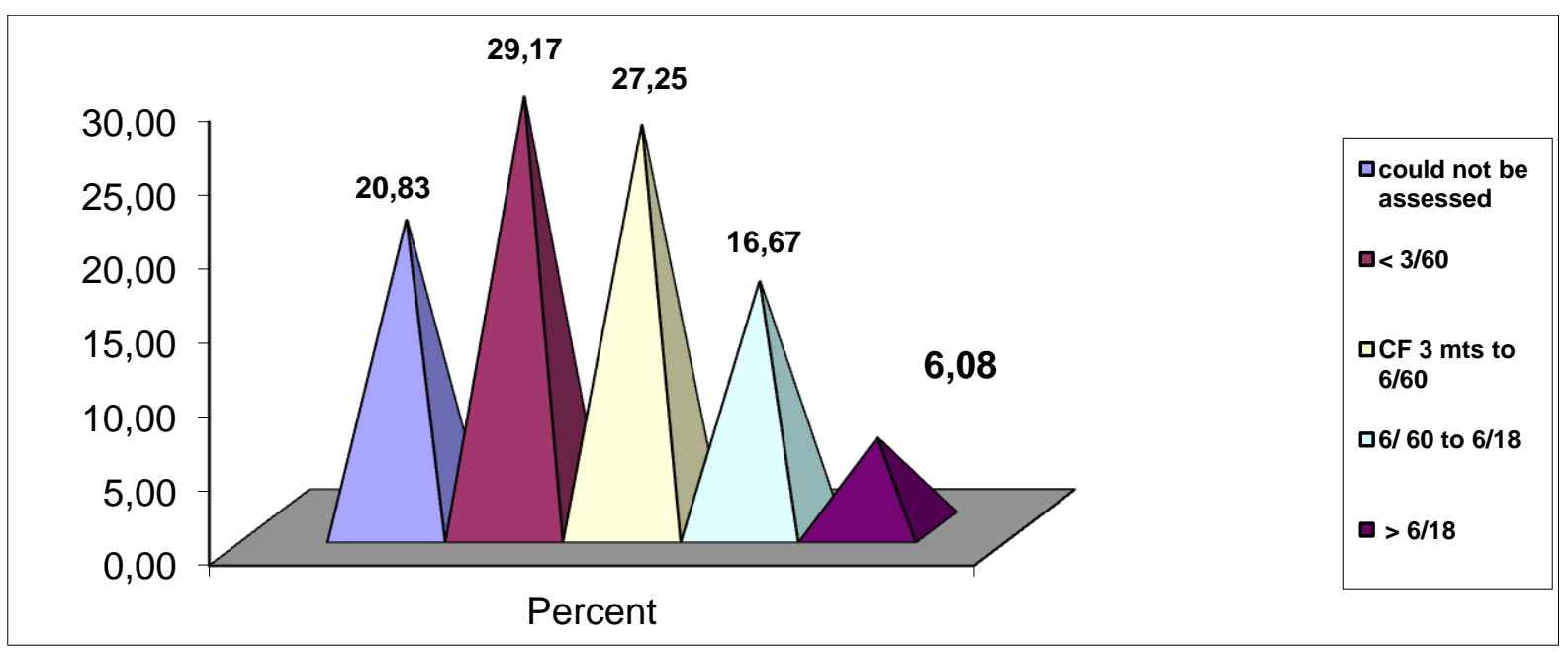

Figure No.14 Post-Operative Visual Acuity of patients in the given sample 
Bibliography

1. Axial eye length growth and final refractive outcome after unilateral paediatric cataract surgery Marije $L$ Sminia1,2, Jan T H N de Faber1, Denise J Doelwijt1,3, René J Wubbels1, Martha Tjon-Fo- Sang1 Bjo.Bmj.com-2010

2. Prediction error and myopic shift after intraocular lens implantation in paediatric cataract patients N E D Hoevenaars1, J R Polling1,2, R C W Wolfs1 Bjo.Bmj.com2011

3. Predictability of formulae for intraocular lens power calculation according to the age of implantation in paediatric cataract ByungJoo Lee1, Sang-Mok Lee2, Jeong Hun Kim1,3, Young Suk Yu3 Bjo.Bmj.com-2019

4. Clinical profile \& visual outcome of traumatic paediatric cataract in suburban Malaysia: a ten-year experience AbdulRahim Adlina, MD, MMed,1 Ying-Jiun Chong, MD,1 and Ismail Shatriah, MD, MMed1 Singapore medical journal,2014ncbi.nlm.nih.gov

5. Challenges in the management of paediatric cataract in a developing country Ifeoma R Ezegwui, Ada E Aghaji, Nkechi $\mathrm{J}$ Uche, and Ernest $\mathrm{N}$ Onwasigwe International journal of Ophthalmology2011,ncbi.nlm.nih.gov

6. Paediatric cataract Reviewed by Jagat Ram P. Nucci, editor. Karger: Basel, Switzerland- Indian journal of medical research-2018-ncbi.nlm.nih.gov

7. The Paediatric Cataract Register (PECARE): an overview of operated childhood cataract in Sweden and Denmark Gunilla Magnusson, Birgitte Haargaard, Saima Basit, Alf Nyström, Annika Rosensvärd, KristinaTornqvist Acta ophthalmologica-2018
8. Predicting future axial length in patients with paediatric cataract and primary intraocular lens implantation Antonio Carlos Lottelli -European journal of Ophthalmology-August,2020-10-05. 